\title{
Relação da Ansiedade com o Zumbido Crônico
}

\section{Relationship of Anxiety with Chronic Tinnitus}

\author{
Mariana Lopes Martins ${ }^{1}$ \\ Cláudia da Silva Carneiro 2 \\ Danilo Augusto de Holanda Ferreira ${ }^{3}$ \\ Wagner Teobaldo Lopes de Andrade ${ }^{4}$ \\ Melyssa Kellyane Cavalcanti Galdino ${ }^{5}$ \\ Thaís Mendonça Maia Wanderley Cruz de Freitas ${ }^{6}$ \\ Marine Raquel Diniz da Rosa ${ }^{7}$
}

\section{RESUMO}

Objetivo: Investigar o nível de ansiedade em pacientes com queixa de zumbido e analisar a relação entre os dois fatores. Metodologia: Pesquisa do tipo estudo de campo, descritiva, transversal e observacional, com caráter quantitativo. A amostra foi composta por 65 indivíduos com queixa de zumbido, sendo 38 mulheres e 27 homens, com média de idades de 53,1 anos e 52,9 anos, respectivamente. Os critérios de elegibilidade foram ter mais de 18 anos e apresentar zumbido crônico. Todos os voluntários passaram por avaliação audiológica básica e realizaram acufenometria. Além disso, realizou-se a anamnese do zumbido e aplicou-se o Tinnitus Handicap Inventory, a Escala Visual Analógica e o Inventário de ansiedade traço e estado. Resultados: Observou-se um nível de ansiedade, predominantemente, baixo na amostra $(50,7 \%$ IDATE-Traço e $76,9 \%$ IDATE-Estado). A intensidade do zumbido não teve relação com o grau de incômodo do som, nem com o nível de ansiedade. Em relação ao incômodo do zumbido, verificou-se que foi proporcional ao aumento da ansiedade traço. Ao dividir os grupos por sexo e por idade e compará-los com o incômodo do zumbido e com os valores do IDATE-Traço, foram encontrados resultados significativos, apenas, para o sexo feminino. Conclusão: Verificou-se uma relação entre o incômodo do zumbido e os níveis de ansiedade. Portanto, a ansiedade deverá ser investigada pelos profissionais que tratam indivíduos com queixa de zumbido, possibilitando a melhoria da qualidade de vida desta população.

\section{DESCRITORES}

Zumbido. Ansiedade. Sintomas Psiquiátricos. Audiologia. Audição. Psicoacústica.

\begin{abstract}
Objective: To investigate the level of anxiety in patients with tinnitus and to analyze the relationship between the two factors. Methodology: a descriptive, cross-sectional, and observational field study, with quantitative character. The sample consisted of 65 individuals with complaints of tinnitus, 38 women and 27 men, with an average age of 53.1 years and 52.9 years, respectively. Eligibility criteria were being over 18 years old and having chronic tinnitus. All volunteers underwent basic audiological evaluation and performed acuphenometry. In addition, the tinnitus case history report, Tinnitus Handicap Inventory, Visual Analogue Scale, State-Trait Anxiety Inventory was performed. Results: There was a predominantly low anxiety level in the sample $(50.7 \%$ STAI - Trait and $76.9 \%$ STAI - State). The intensity of tinnitus is not related to the degree of discomfort of the sound or the level of anxiety. However, the tinnitus annoyance is proportional to the increase of anxiety trait. By dividing the groups by gender and age and comparing the tinnitus discomfort with the STAI-Trait values, significant results were found only for women. Conclusion: There is a relationship between tinnitus discomfort and anxiety levels, therefore anxiety should be investigated by professionals who treat individuals with tinnitus in order to improve the quality of life of this population.
\end{abstract}

\section{DESCRIPTORS}

Tinnitus. Anxiety. Psychiatric Symptoms. Audiology. Hearing. Psychoacoustics.

${ }^{1}$ Mestre em Neurociências, Professora Substituta, Departamento de Fonoaudiologia, Universidade Federal da Paraíba. João Pessoa, PB, Brasil.

${ }^{2}$ Mestre em Neurociências, Fonoaudióloga, Departamento de Fonoaudiologia, Universidade Federal da Paraíba. João Pessoa, PB, Brasil. ${ }^{3}$ Mestre em Odontologia Preventiva e Infantil, Professor, Instituto Federal de Educação, Ciência e Tecnologia da Paraíba. Patos, PB, Brasil. ${ }^{4}$ Doutor em Linguística, Professor Adjunto, Departamento de Fonoaudiologia, Universidade Federal da Paraíba. João Pessoa, PB, Brasil. ${ }^{5}$ Doutora em Neuropsiquiatria e Ciências do Comportamento, Professora Adjunta, Departamento de Psicologia, Universidade Federal da Paraíba. João Pessoa, PB, Brasil.

${ }^{6}$ Mestranda em Fonoaudiologia, Programa de Pós-graduação em Fonoaudiologia, Departamento de Fonoaudiologia, Universidade Federal da Paraíba. João Pessoa, PB, Brasil.

${ }^{7}$ Doutora em Farmacologia, Professora Adjunta, Departamento de Fonoaudiologia, Universidade Federal da Paraíba. João Pessoa, PB, Brasil. 
zumbido é uma sensação auditiva anômala ${ }^{1}$, caracterizado pela percepção de som na ausência de estímulo sonoro externo. A prevalência é de 10 a $15 \%$ da população mundial ${ }^{2}$. Quando o zumbido vem relacionado à comorbidades psicológicas, pode ser classificado como compensatório ou não compensatório. O zumbido é definido como compensatório quando a pessoa não se sente afetado por ele, ou só se sente prejudicado em algumas situações específicas; enquanto, o não compensatório, a pessoa acometida sofre consideravelmente em todas as esferas da vida e está associado ao desenvolvimento de sintomas secundários, como problemas para adormecer, dificuldades de concentração, ansiedade e depressão ${ }^{3}$.

O zumbido pode ser decorrente de diversas patologias ${ }^{4}$ e alguns fatores podem agravá-lo, como o estresse, a ansiedade e a depressão ${ }^{1}$; tais fatores se tornam potencializadores do incômodo em indivíduos com zumbido ${ }^{5}$.

A ansiedade inclui distúrbios como o medo excessivo e demais perturbações comportamentais relacionadas. Ocorre a antecipação de uma ameaça futura, com picos de excitação necessários para lutar ou fugir, pensamentos de perigo imediato, bem como o aumento da tensão muscular e do estado de vigília ${ }^{6}$.

A ansiedade patológica é diferente de medo e de ansiedade transitória. A ansiedade patológica é muitas vezes induzida pelo estresse e, quando persistente e em níveis elevados, pode gerar prejuízo ocupacional, social e nas relações interpessoais, sendo característico dos transtornos ansiosos $^{6}$.

Segundo o Diagnostic and Statistical Manual of Mental Disorders (DSM-V), os transtornos ansiosos podem ser classificados em transtorno de ansiedade de separação, mutismo seletivo, fobia específica, transtorno de ansiedade social (fobia social), transtorno do pânico, ataques de pânico específico, agorafobia, distúrbio de ansiedade generalizada, transtorno de ansiedade induzida por medicação ou por outra condição médica, transtorno de ansiedade especificado e não especificado ${ }^{6}$.

Para relacionar a etiologia do zumbido com a adaptação fisiológica da ansiedade, como um processo psicologicamente determinado, foi criado o modelo psicofisiológico de McKenna et $\mathrm{al}^{7}$ (Figura 1), baseado no modelo neurofisiológico de Jastreboff ${ }^{4}$, que tem como mediador o disparo do medo e a geração de desordens de ansiedade para manutenção da percepção do zumbido.

Segundo o modelo psicofisiológico, o zumbido provoca angústia quando o indivíduo detém pensamentos excessivamente negativos e constantes sobre ele. Esses pensamentos provocam a manutenção de fatores psicológicos negativos, que resultam na mudança da detecção do zumbido, isto é, os indivíduos obtêm uma percepção distorcida do timbre, tom e/ou volume do zumbido ${ }^{7}$.

Algumas hipóteses formuladas para a compreensão da relação entre o zumbido e a ansiedade são: 1. A ansiedade pode ser pré-existente e produtora de estressores que exacerbam ou pioram o zumbido; ; 2 . 0 zumbido pode ser uma das causas da ansiedade ${ }^{8}$ ou ainda 3. Pode existir uma predisposição individual de pessoas com zumbido a serem mais ansiosas ${ }^{7,9}$.

O objetivo do presente trabalho foi investigar o nível de ansiedade em pacientes com queixa de zumbido e analisar a relação entre os dois fatores.

\section{METODOLOGIA}

O presente estudo foi conduzido de acordo 
Figura 1. Tradução para o português do modelo cognitivo de sofrimento do zumbido.

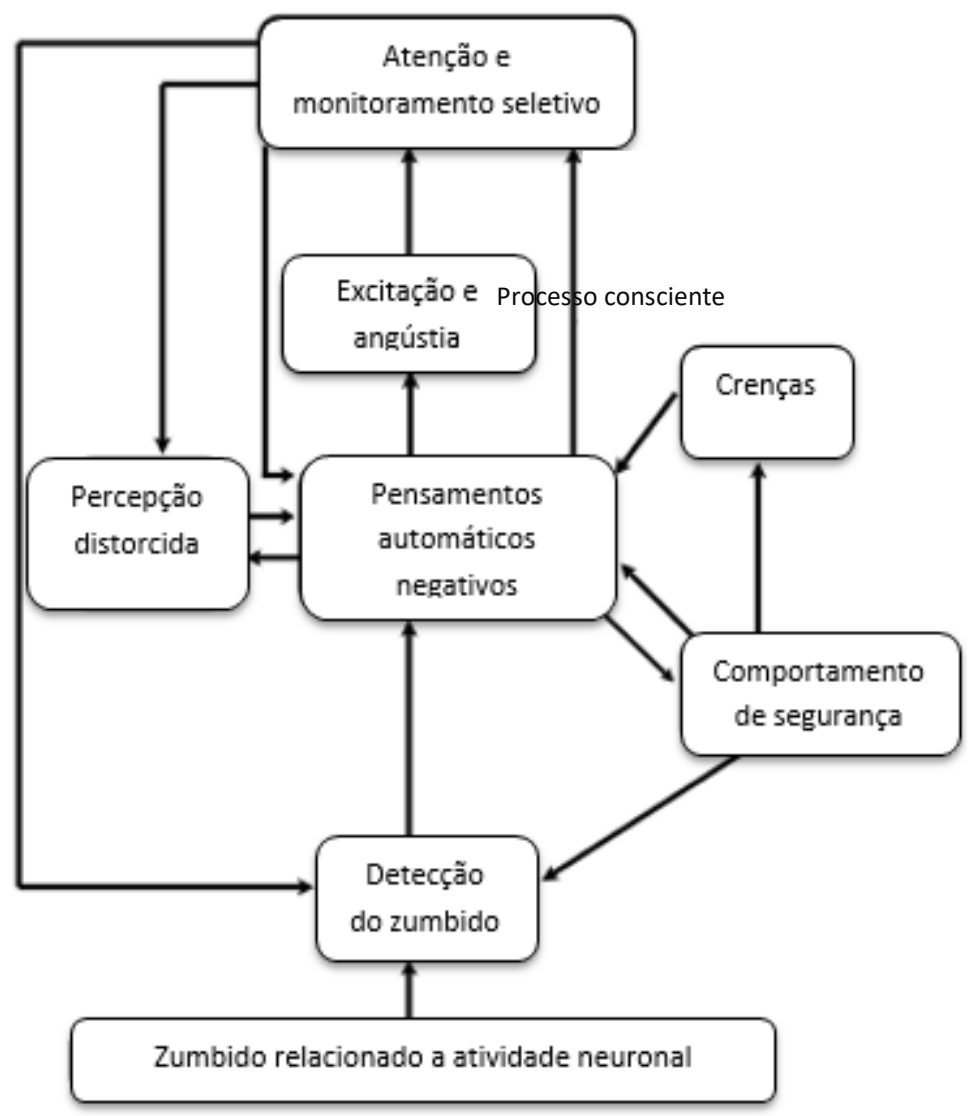

com os princípios éticos da Declaração de Helsinki. Os protocolos experimentais foram aprovados pelo Comitê de Ėtica em Pesquisas (CAAE: 029539126.0000.5188). Consentimento informado foi obtido de todos os participantes.

Tratou-se de uma pesquisa de estudo de campo do tipo descritiva, transversal e observacional, com caráter quantitativo. A amostra se deu por conveniência, formada por 65 voluntários com queixa de zumbido, sendo 38 mulheres e 27 homens, com média de idades de 53,1 anos e 52,9 anos, respectivamente.

Foram incluídos no estudo os voluntários que aceitaram participar da pesquisa e que procuraram voluntariamente o setor de atendimento ao indivíduo com zumbido. Pelo que estes voluntários deveriam se enquadrar nos critérios de elegibilidade seguintes: ter mais de 18 anos e apresentar zumbido crônico. Foi diagnosticado como zumbido crônico, quando tal esteve presente a mais de seis meses, com base em critérios previamente publicados por Shulman e Farhadi ${ }^{1,10}$.

Após assinarem o Termo de consentimento livre e esclarecido (TCLE), foram submetidos, primeiramente, à avaliação audiológica básica e acufenometria. Além disso, aplicou-se anamnese específica de zumbido, Tinnitus Handicap Inventory (THI), Escala visual analógica (EVA), Inventário de ansiedade traço (IDATE- traço) e Inventário de ansiedade estado (IDATE-estado). 
Foi realizada audiometria tonal, com o objetivo de mensurar o limiar auditivo para que, posteriormente, este fosse utilizado como base para a acufenometria. Foram pesquisados os limiares auditivos nas vias aéreas e ósseas, no espectro de frequência de 125 a $8000 \mathrm{~Hz}$ na via aérea e 500 a $4000 \mathrm{~Hz}$ na via óssea ${ }^{11}$. Foi utilizado audiômetro de dois canais (Modelo: AVS 500 e Marca: Vibrasom), em cabina acústica.

A acufenometria, também conhecida como medidas psicoacústicas do zumbido, mensura a sensação de intensidade (loudness) e a sensação de frequência (pitch) do som percebido pelo paciente ${ }^{12-13}$. De forma que foi apresentado o som na orelha contralateral, em caso de zumbido unilateral, ou apresentado na melhor orelha, no caso de zumbido bilateral. $\mathrm{O}$ tom foi variado e foi perguntado ao paciente qual som se assemelhava mais com o seu zumbido ${ }^{11}$. Após a escolha do som, foram apresentadas frequências que variavam de 125 a 8000 Hertz, em uma intensidade acima do limiar audiométrico encontrado ${ }^{12,14}$. Posteriormente foi analisada a intensidade, variando-a e ajustando-a até chegar ao mesmo nível do zumbido ouvido pelo indivíduo. Foi possível variar a intensidade em passos de um decibel ${ }^{12}$.

A anamnese específica do zumbido tem como objetivo principal coletar dados do zumbido sob a perspectiva do paciente, assim tem-se dados gerais, como a localização; tempo em que o zumbido está presente; surgimento do zumbido; tipo de zumbido: único, múltiplo ou pulsátil; características acústicas parecidas com o som ouvido; intensidade em que o som é percebido; relação do zumbido com o incômodo na vida do paciente e, por fim, a causa do zumbido.
O THI é uma medida de autorrelato a fim de quantificar o impacto do zumbido na vida diária, criado por Newman, Jacobson e Spitzer (1996) e adaptado à população brasileira por Ferreira (2005). O questionário é composto por 25 questões e agrupado em três subescalas: funcional, emocional e catastrófica. As respostas podem variar em "sim", "não" ou "às vezes", e cada pergunta se relaciona com um dos três domínios subescalares: funcional, emocional ou catastrófico ${ }^{15}$. Os resultados do THI podem ser classificados em cinco graus: discreto; leve; moderado; severo e catastrófico ${ }^{16}$.

A Escala visual analógica (EVA) é utilizada para quantificar a dor crônica e consiste em uma forma gráfico-visual para determinar o desconforto gerado pelo zumbido, medido em uma escala de 0 a $10^{17}$.

O Inventário de ansiedade traço-estado (IDATE) baseia-se na concepção de ansiedade proposta por Spielberger (1977) ${ }^{18}$ e validada para o português por Biaggio, Natalício e Spielberguer (1997) ${ }^{19}$, em que o IDATE-Estado é caracterizado como um estado emocional transitório com sentimentos desagradáveis de apreensão, tensão, nervosismo ou preocupação ${ }^{18-19}$. O indivíduo responde de acordo com a ansiedade que está sentindo no momento da aplicação do teste.

Enquanto, o IDATE-Traço relaciona-se às diferenças comportamentais de cada indivíduo conforme foram adquiridas ${ }^{18-19}$; neste inventário, cada paciente responde como se sente habitualmente, a partir das suas características individuais relativamente estáveis que predispõem à ansiedade, ou seja, a partir das suas reações a situações ameaçadoras. Existem 20 itens para cada um dos questioná- 
rios IDATE Traço e Estado, com quatro graus de intensidade cada9,18-19.

Por fim, os dados coletados foram analisados quantitativamente e digitados em uma planilha no Microsoft Excel, versão 2003, para constituir o banco de dados que atende à demanda desta pesquisa. A partir deste, foram extraídos os resultados expressos por meio de quadros e tabelas (Técnica de estatística descritiva). Para realizar a análise Inferencial, inicialmente, foi realizado o teste de normalidade, Kolmogorov-Smirnov e, a partir daí, estabelecidos os testes ANOVA one-way e o teste de correlação de Pearson. Para as variáveis categóricas, foi utilizado o teste qui-quadrado. As diferenças foram consideradas significativas quando apresentaram um nível de significância de $5 \%(p<0,05)$. A análise estatística foi realizada por meio do software Statistic Package for Social Sciences (SPSS) 24.

\section{RESULTADOS}

Foi observada maior ocorrência do zumbido contínuo em ambas as orelhas, com intensidade média, audível o tempo inteiro, com surgimento repentino e presente há anos. Além disso, a maioria dos indivíduos se incomoda com o sintoma (Tabela 1).

Tabela 1. Dados do questionário específico de zumbido em porcentagem.

\begin{tabular}{lll}
\hline Variáveis & $\mathrm{n}$ & $\%$ \\
\hline Localização & & \\
Orelha Direita & 15 & 23 \\
Orelha Esquerda & 12 & 18 \\
Ambas as orelhas & 29 & 45 \\
Na cabeça & 09 & 14 \\
Tempo & & \\
Meses & 11 & 17 \\
Anos & 54 & 83 \\
Surgimento & & \\
Repentino & 38 & 58 \\
Gradual & 24 & 37 \\
Após a exposição a ruído & 02 & 03 \\
Não identifica & 01 & 02 \\
Tipo & & \\
Contínuo & 61 & 94 \\
Pulsátil & 03 & 05 \\
Intermitente & 01 & 02 \\
Período & & \\
Tempo Inteiro & 39 & 60 \\
Oscilante & 07 & 11 \\
Um período & 07 & 11 \\
Ao deitar-se & 09 & 14 \\
No silêncio & 03 & 05 \\
Altura & & \\
Baixo & 15 & 23 \\
Médio & 31 to & 54 \\
Incômodo & 15 & 23 \\
Sim & & \\
Não & 38 & 58 \\
\hline
\end{tabular}


Ao mensurar a sensação de frequência (pitch) e a intensidade (loudness) do zumbido, por meio da acufenometria, foi observada a moda de $12,30 \%$, em $50 \mathrm{~dB}$ e frequência de $6000 \mathrm{~Hz}$.

Na EVA, obteve-se a média de $5,83 \pm 2,92$, de forma que a resposta mais recorrente $(18,40 \%)$ foi nas intensidades de 6 e 10.

Dentre os graus de incômodo do zumbido, utilizando-se o questionário $\mathrm{THI}$, observou-se que grande parte apresentou o grau leve. Em relação aos domínios do THI, o catastrófico foi o que mais esteve presente, seguido do domínio emocional (Tabela 2).

Ao mensurar o nível de ansiedade, foram observadas percentagens de 50,70\% $(n=33)$ para baixa ansiedade, $40,00 \%(n=26)$ com média ansiedade, $9,20 \%(n=6)$ para alta ansiedade no questionário IDATE-Traço. No questionário IDATE-Estado foi observado, $76,90 \%$ ( $n=50$ ) com baixa ansiedade, $20,00 \%$ $(n=13)$ para média ansiedade e 3,00\% $(n=2)$ com alta ansiedade. Com isso, percebe-se na amostra pesquisada que a maior parte das respostas se refere à ocorrência de ansiedade baixa.

Ao comparar a intensidade do zumbido (acufenometria) com o grau de incômodo (THI), não foram observados resultados significativos $(p=0,358)$. Desta forma, não foi observada uma relação entre a intensidade do zumbido e o seu grau de incômodo na população pesquisada (Tabela 3).

O coeficiente de correlação da sensação de intensidade (loudness) da acufenometria com o IDATE Estado não foi significativo ( $r$ $=-0,010 ; p=0,939)$ (Figura 2). Desse modo,

Tabela 2. Descrição estatística dos escores (\%) obtidos no Tinnitus Handicap Inventory em cada domínio.

\begin{tabular}{|c|c|c|c|}
\hline \multirow{2}{*}{$\begin{array}{c}\text { Valores } \\
(\%)\end{array}$} & \multicolumn{3}{|c|}{ Domínios } \\
\hline & Funcional & Emocional & Catastrófico \\
\hline Média & 35,17 & 39,31 & 47,53 \\
\hline Erro padrão & 3,16 & 4,00 & 3,21 \\
\hline
\end{tabular}

Tabela 3. Quantidade de indivíduos classificados quanto ao grau de incômodo do zumbido e médias da intensidade na acufenometria em cada agrupamento.

\begin{tabular}{l|c|c|c|cc}
\hline \multirow{2}{*}{ Grau THI } & \multirow{2}{*}{$\begin{array}{c}\text { Intervalo do } \\
\text { escore THI }\end{array}$} & $\mathrm{n}$ & $\%$ & \multicolumn{2}{c}{$\begin{array}{c}\text { Acufenometria } \\
\text { Intensidade (dBNA) }\end{array}$} \\
\cline { 5 - 7 } & & & & Média & Erro padrão \\
\hline Discreto & $0-16$ & 14 & 21,50 & 46,36 & 4,55 \\
Leve & $18-36$ & 22 & 33,80 & 45,34 & 4,74 \\
Moderado & $38-56$ & 13 & 20,00 & 55,50 & 5,10 \\
Severo & $58-76$ & 07 & 10,80 & 43,50 & 6,80 \\
Catastrófico & $78-100$ & 09 & 13,80 & 56,56 & 5,58 \\
TOTAL & $0-100$ & 65 & 100,00 & - & - \\
\hline
\end{tabular}


Figura 2. Correlação entre intensidade da acufenometria e inventário de ansiedade transitória.

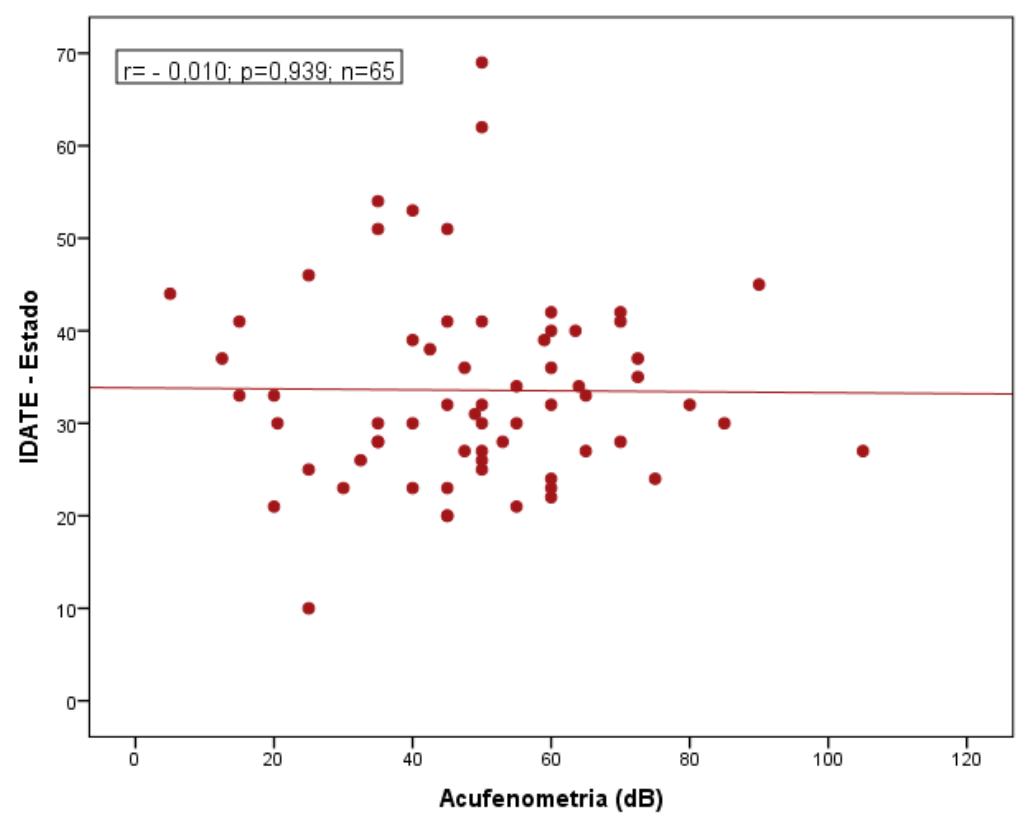

Tabela 4. Classificações do Inventário de Ansiedade traço correlacionados com as classificações do Tinnitus Handicap Inventory.

\begin{tabular}{c|cccccc|c|c}
\hline \multirow{2}{*}{$\begin{array}{c}\text { IDATE Traço } \\
\text { Classificação }\end{array}$} & \multicolumn{6}{c|}{ Classificações THI (\%) } & \multirow{2}{*}{ Total } \\
\cline { 2 - 6 } & Discreto & Leve & Moderado & Severo & Catastrófico & \multirow{2}{*}{ Baixo } \\
\multirow{2}{*}{ Médio } & 08 & 11 & 04 & 05 & 02 & 30 \\
& 26,70 & 36,70 & 13,30 & $16,70 \%$ & $6,70 \%$ & 100,00 \\
\hline \multirow{2}{*}{ Alto } & 05 & 10 & 08 & - & 03 & 26 \\
& 19,20 & 38,50 & 30,8 & - & 11,50 & 100,00 \\
\hline \multirow{2}{*}{ Total } & - & - & - & 02 & 04 & 06 \\
& - & - & - & 33,30 & 66,70 & 100,00 \\
\hline & 13 & 21 & 12 & 07 & 09 & 62 \\
& 21,00 & 33,90 & 19,40 & 11,30 & 14,50 & 100,00 \\
\hline
\end{tabular}

a sensação de intensidade do zumbido não foi relacionada com o nível de ansiedade no momento do teste.

Ocorreu associação significativa $(p=0,001)$ entre o nível de ansiedade (IDATE Traço) e o grau de incômodo do zumbido, medido por meio do THI (Tabela 4), existindo um aumento proporcional do incômodo do zumbido, a partir do aumento da ansiedade e vice-versa. Foi observado predomínio dos graus de incômodo: "discreto" e "leve" em voluntários com níveis baixos de ansiedade; "leve" e "moderado" nos indivíduos com níveis médios de ansiedade; e predominando 
Figura 3. Correlação da escala visual analógica e inventário de ansiedade traço.

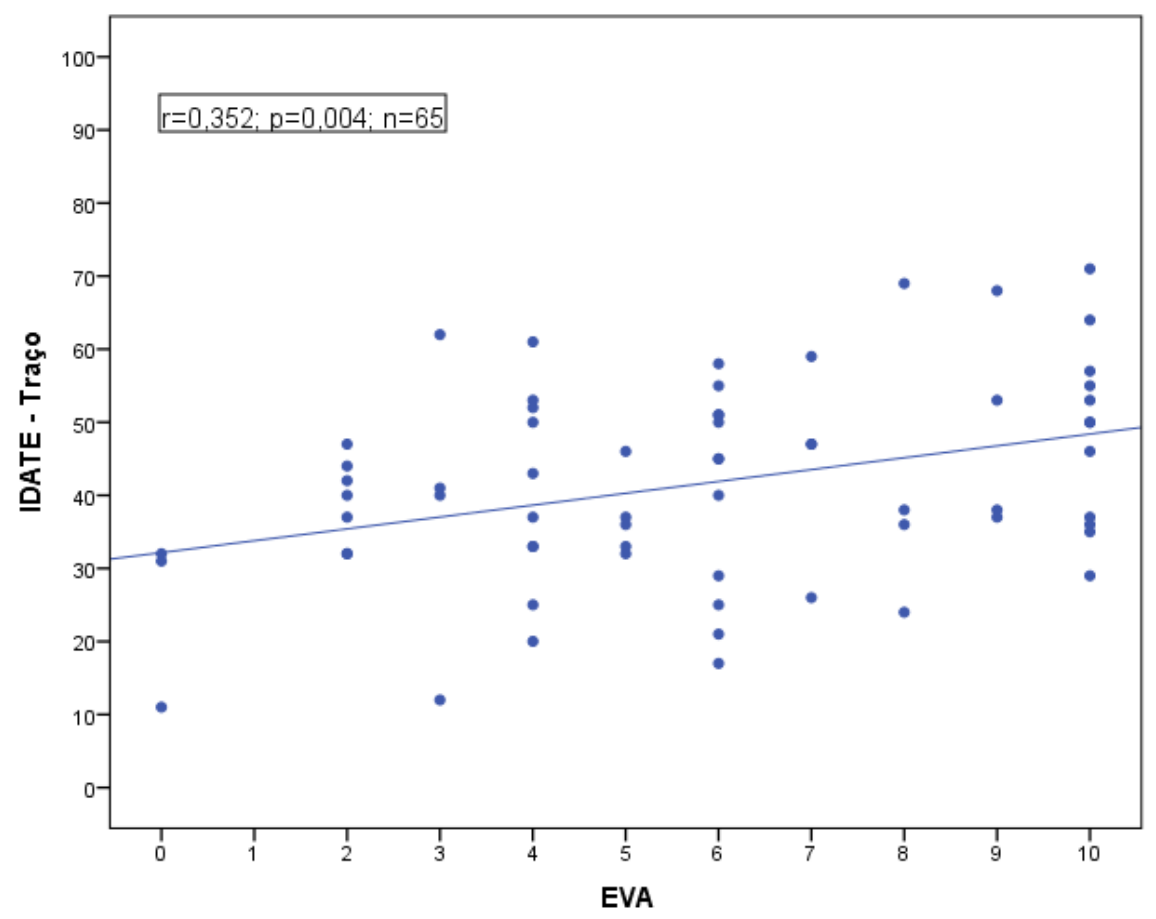

as respostas em "severo" e "catastrófico" em indivíduos com alta ansiedade.

Ao se correlacionar o THI com o IDATE - Traço, controlando o efeito da variável "idade" para cada sexo separadamente, observou-se que existiu uma correlação significativa apenas para o sexo feminino $(r=0,536$; $p=0,001)$, não ocorrendo para os homens $(r=0,132 ; p=0,504)$.

Comparando-se entre os sexos os valores do IDATE - Traço, observou-se que a média para o sexo masculino foi de $39,97 \pm 13,34$ pontos e para o feminino foi de $42,97 \pm 13,60$ pontos. No entanto, essa diferença não foi considerada significativa $(p=0,375)$. Ao comparar com a idade, não foi observada correlação significativa entre os níveis de ansiedade através do IDATE Traço e a idade dos participantes do estudo $(r=-0,080 ; p=0,526)$.
Em relação aos escores obtidos na EVA e no IDATE - Traço, ocorreu uma correlação significativa $(r=0,352 ; p=0,004)$ (Figura 3).

\section{DISCUSSÃO}

Observou-se o aumento do incômodo do zumbido proporcionalmente ao aumento da ansiedade, mesmo a maioria dos participantes tendo apresentando baixos níveis de ansiedade, conforme foi apontado em demais estudos $^{20}$. Este fato ocorre em face dos indivíduos com zumbido apresentarem aumento da atividade neural no córtex auditivo esquerdo e uma diminuição da atividade neural no sistema límbico (hipocampo e amígdala), ocorrendo comunicação disfuncional entre estas regiões cerebrais ${ }^{21}$. Assim como, ocorre uma conectividade direcional do nucleus accumbens, no sistema límbico, para o córtex pré-frontal 
e essa conectividade é associada à duração do zumbido ${ }^{22}$.

O estudo de Besteher et al. ${ }^{23}$ mostra que indivíduos com zumbido apresentam redução da substância cinzenta para-hipocampal esquerda e a ansiedade apresentou-se como potencialmente moduladora da atividade cerebral nestes indivíduos. Assim, o estudo concluiu que a ansiedade e a depressão são os principais moduladores dos efeitos estruturais do cérebro no zumbido.

Ao comparar os indivíduos com zumbido e a população de forma geral, foi observado que os pacientes com zumbido têm uma taxa de sintomas psiquiátricos maior que a população ${ }^{8,24,25}$. Além disso, a severidade do zumbido pode ser predita pelos sintomas da ansiedade ${ }^{25}$ e, os indivíduos com zumbido, têm maiores chances de manifestarem transtornos de ansiedade ${ }^{24}$.

Em relação ao sexo, existe uma associação entre o incômodo do zumbido e a ansiedade no sexo feminino, não ocorrendo no sexo masculino, o que também já foi observado por outros autores ${ }^{24}$. O fato deve-se ao aumento da ativação cerebral no córtex orbitofrontal, ínsula, córtex cingulado anterior, para-hipocampo e o córtex auditivo, que são regiões importantes para o processamento emocional dos sons ${ }^{26}$.

Além disso, Andersson et al. ${ }^{25}$ verificaram uma significativa correlação entre a sensibilidade ansiógena do indivíduo e o grau de gravidade do zumbido, verificando que este aspecto é mais marcante nas mulheres ${ }^{27}$. Van der Wal et al. ${ }^{28}$ identificaram que as mulheres apresentam níveis mais elevados de incômodo e de ansiedade quando comparado aos homens. Porém, outras pesquisas mostram não existir relação significativa entre o sexo e a severidade do zumbido $20,29-30$.

Acredita-se que as redes associadas à atenção, memória, angústia e experiência multissensorial são envolvidos com a percepção do zumbido ${ }^{31}$. Essa percepção pode ser modulada pelo córtex pré-frontal dorsolateral, que desempenha um papel importante na atenção, no sistema límbico e no córtex auditivo secundário ${ }^{32}$. Assim, o fato dos idosos ficarem mais tempo em casa, no silêncio e apresentarem maior atenção auditiva ao zumbido ${ }^{29}$, podem apresentar, também, maior incômodo do zumbido $24,30,33$.

Existem modelos que comprovam que a atenção é um fator que contribui para o aumento e a manutenção do zumbido ${ }^{34}$, que supõe que existe uma disparidade entre o que o cérebro prevê que ele deva ouvir (essa expectativa influenciada pela atividade neural subjacente à percepção do zumbido) e as informações acústicas que são entregues pelo ouvido ao cérebro ${ }^{35}$. Os idosos têm mais dificuldade de adaptação ao zumbido e essa dificuldade de habituação pode ser influenciada por fatores psicológicos ${ }^{33}$. Entretanto, na presente pesquisa, a idade não teve influência na ansiedade, não existindo relação, para ambos os sexos, entre a ansiedade e a idade da amostra pesquisada. Corroborando com outros estudos que também não encontraram relação entre a idade dos participantes e o incômodo do zumbido ${ }^{20,29}$.

O aumento dos valores do grau de incômodo no THI ocorreu na mesma proporção dos valores da EVA, sugerindo que tais instrumentos podem ser utilizados associadamente. Os dados do THI e da EVA apresentam maior relevância e confiabilidade quando são correlacionados ${ }^{16}$, visto que fornecem dimensões 
diferentes do zumbido e se complementam ${ }^{36}$.

A literatura também refere correlação entre o zumbido e a ansiedade ${ }^{37}$. Dentre os pacientes com zumbido, de $15 \%$ a $90 \%$ deles apresentam ansiedade como um dos sintomas $^{5,22}$, ocorrendo em 19\% (aproximadamente) a ansiedade, de moderada a severa ${ }^{37}$. Ou, muitas vezes, mais de $50 \%$ da população com zumbido estudada apresenta sintomatologia ansiosa, com percentagem igual para os que apresentavam quadro depressivo ${ }^{38}$.

Não foi observada relação significativa entre a sensação de intensidade do zumbido, o nível de incômodo e de ansiedade, porém, as pessoas que apresentaram níveis altos de ansiedade relataram perceber intensidade superior às pessoas que apresentavam níveis baixos de ansiedade, o que corrobora com a literatura prévia sobre o tema, pois esta afirma que quando zumbido e fatores emocionais significativos estão presentes (como a ansiedade), ocorrendo a amplificação da percepção do zumbido através da criação de um feedback positivo dentro das redes corticais ${ }^{4}$.

Estes resultados sugerem a explicação para que no autorrelato: o zumbido pode ser classificado como extremamente alto, mesmo quando a medida psicoacústica sugerir o contrário. A associação com a angústia leva a este aumento na sensação de intensidade maior. Desta forma, quando se tem o aumento na sensação da intensidade do som, consequentemente, se tem o aumento dos pensamentos automáticos negativos ${ }^{7}$.

O tratamento da ansiedade ou da depressão é considerado crítico para o sucesso de toda e qualquer tentativa de tratar o componente sensorial ${ }^{1}$. Assim, o indivíduo que tiver fatores psicológicos negativos associados deve ser encaminhado para tratamento psicológico e/ou psiquiátrico, a fim de corrigir esses pensamentos automáticos negativos; reduzir a atividade do sistema nervoso autônomo simpático; reduzir a atenção seletiva e o monitoramento de sinais ao zumbido; corrigir as percepções distorcidas de intensidade do zumbido e o seu impacto no funcionamento; corrigir os comportamentos de segurança contraproducentes e crenças imprecisas ${ }^{1,7}$.

A terapia cognitiva comportamental tem mostrado eficácia no alívio da angústia causada pelo zumbido. Porém, geralmente não reduz a intensidade do zumbido ${ }^{39}$. Estas intervenções não modificam as características iniciais do zumbido, mas buscam reorganizar as funções mentais alteradas, mudando o significado psicológico do zumbido e como resultado, o zumbido torna-se menos ameaçador e invasivo, ocorrendo mudanças nas associações do sintoma com estados emocionais positivos ${ }^{4}$.

\section{CONCLUSÃO}

Ocorreu a presença de ansiedade, predominantemente, de grau leve nos pacientes com zumbido, além de relação entre o incômodo do zumbido e os níveis de ansiedade, sendo assim, importante e necessária a investigação e a atenção do sintoma pelos profissionais que tratam indivíduos com queixa de zumbido, visando a melhoria da qualidade de vida desta população. 


\section{REFERÊNCIAS}

1. Shulman A, Goldstein B. Subjective Idiopathic Tinnitus and Palliative Care: A Plan for Diagnosis and Treatment. Otolaryngol Clin. 2009; 15-37.

2. Gopinath B, Mcmahon CM, Rochtchina E, Karpa MJ, Mitchell P. Incidence, Persistence, and Progression of Tinnitus Symptoms in Older Adults: The Blue Mountains Hearing Study. Ear \& Hearing. 2010; 31:407-412.

3. Stobik C, Weber RK, Munte TR, Wolter M, Frowry J. Evidence of psychosomatic inferences in compensatory and no compensatory tinnitus. International $j$ of audiology. 2005; 44:370-378.

4. Jastreboff PJ. Phantom auditory perception (tinnitus): mechanisms of generation and perception. Neuroscience Researc. 1990; 8:221-254

5. Dobie RA. Depression and tinnitus. The Otolaryngologic Clinics of North America. 2003; 36:383-388.

6. American Psichiatric association. Diagnostic and Statistical Manual of Mental Disorders. $5^{\mathrm{a}}$.ed. Arlington, VA: American Psychiatric Publishing; 2013.

7. Mckenna L, Handscomb L, Hoare DJ, Hall DA. A scientific cognitive-behavioral model of tinnitus: novel conceptualizations of tinnitus distress. Frontiers in neurology. 2014; 5:1 -15

8. Kaltenbach JA. The dorsal cochlear nucleus as a participant in the auditory, attentional and emotional components of tinnitus. Hearing Research. 2006; 224-234.

9. Gül Al, Özkiriş M, Aydin R, Simşek G, Saydam L. Coexistence of anxiety sensitivity and psychiatric comorbidities in patients with chronic tinnitus. Neuropsychiatric Disease and Treatment. 2015; 11:413-418.

10. Farhadi M, Mahmoudian S, Saddadi F, Karimian AR, Mirzaee M, Ahmadizadeh M, et al. Functional brain abnormalities localized in 55 chronic tinnitus patients: Fusion of SPECT coincidence imaging and MRI. Journal of Cerebral Blood Flow and Metabolism. 2010; 30:864-870.

11. Oliveira PM. Avaliação audiológica do zumbido. In: Figueiredo RR, Azevedo AA. Zumbido. Revinter; 2013.

12. Branco FCA. Avaliação Audiológica Básica e Psicoacústica do Zumbido. In A.G. Samelli. Zumbido: Avaliação, Diagnóstico e Reabilitação. São Paulo: Lovise. 2004; 57-58.

13. Kostek B, Poremski T. A new method for measuring the psychoacoustical properties of tinnitus. Diagnostic Pathology. 2013; 8:209-222.

14. Henry JA, McMillan GP, Thielman EJ, Galvez G, Zaugg TL, Porsov E, et al. Evaluating psychoacoustic measures for establishing presence of tinnitus. Journal of Rehabilitation Research \& Development. 2013; 50(4):573-584.

15. Fioretti AB, Fusetti M, Eibenstein A. Association between sleep disorders, hyperacusis and tinnitus: Evaluation with tinnitus questionnaires. Noise Health. 2013; 15:91-95.
16. Figueiredo RR, Azevedo AA, Oliveira PM. Análise da correlação entre a escala visual-análoga e o Tinnitus Handicap Inventory na avaliação de pacientes com zumbido. Rev Bras Otorrinolaringologia. 2009; 75:76-79.

17. Azevedo AA, Oliveira PM, Siqueira AG, Figueiredo RR. Análise crítica dos métodos de mensuração do zumbido. Rev Bras Otorrinolaringologia. 2007; 73:418-423.

18. Spielberger CD. The State-Trait Anxiety Inventory. 1977.

19. Biaggio AMB, Natalício L, Spielberguer CD. Desenvolvimento da forma experimental em português do Inventário de Ansiedade Traço-Estado (IDATE). Arq bras Psicapl. 1997; 29, 31-44

20. Udupi VA, Uppunda AK, Mohan KM, Alex J, Mahendra $\mathrm{MH}$. The relationship of perceived severity of tinnitus with depression, anxiety, hearing status, age and gender in individuals with tinnitus. International Tinnitus Journal. 2013; 18:29-34.

21. Qu T, Qi T, Yu S, Wei W, Cai A, Wang J, et al. Dynamic Changes of Functional Neuronal Activities Between the Auditory Pathway and Limbic Systems Contribute to Noise-Induced Tinnitus with a Normal Audiogram. Neuroscience. 2019; 408:31-45

22. Xu J, Cui J, Feng $Y$, Yong $W$, Chen $\mathrm{H}$, Chen $\mathrm{Y}$, Yin $\mathrm{X}$ Wu Y. Chronic Tinnitus Exhibits Bidirectional Functional Dysconnectivity in Frontostriatal Circuit. Front. Neurosci. 2019; 13:1299.

23. Besteher B, Gaser C, Ivanšić D, Guntinas-Lichius O, Dobel C, Nenadić I. Chronic tinnitus and the limbic system: Reappraising brain structural effects of distress and affective symptoms. Neurolmage: Clinical. 2019; 24:101976.

24. Lin C, Chen L, Chou P, Chung C. Increased prevalence and risk of anxiety disorders in adults with tinnitus: $A$ population-based study in Taiwan. 2018; 50:131-136.

25. Strumila R, Lengvenyte A, Vainutiene V, Lesinskas E. Influence of personality traits on tinnitus severity perception, anxiety and depressive symptoms. PsychiatrQ. 2017; 88(4): 865-877.

26. Vanneste S, Joos K, De Ridder D. Prefrontal Cortex Based Sex Differences in Tinnitus Perception: Same Tinnitus Intensity, Same Tinnitus Distress, Different Mood. PLoS ONE. 2012; 7(2): e31182.

27. Andersson G, Vretblad P. Larsen H-C, Lyttkens L. Longitudinal follow-up of tinnitus complaints. Archives of Otolaryngology, Head and Neck Surgery. 2001; 127:175-179.

28. Van der Wal A, Luyten $\mathrm{T}$, Cardon E, Jacquemin L, Vanderveken OM, Topsakal V, Van de Heyning P, De Hertogh W, Van Looveren N, Van Rompaey V, Michiels S and Gilles A. Sex Differences in the Response to Different Tinnitus Treatment. Front Neurosci. 2020; 14:422.

29. Pinto PCL, Sanchez TG, Tomita S. Avaliação da relação entre severidade do zumbido e perda auditiva, sexo e idade do paciente. Braz J Otorhinolaryngol. 2010; 76:18-24 
30. Gibrin PCD, Ciquinato DSA, Gonçalves IC, Marchiori VM, Marchiori LLM. O zumbido e sua relação com ansiedade e depressão em idosos: uma revisão sistemática. Rev. CEFAC. 2019; 21(4):7918.

31. Walpurger V, Hebing-lennartz G, Denecke H, Pietrowsky R. Habituation deficit in auditory event-related potentials in tinnitus complainers. Hear Res. 2003; 181:57-64.

32. Mirz F, Gjedde A, Ishizu K, Pedersen CB. Cortical networks subserving the perception of tinnitus - a PET study. Acta Otolaryngol Suppl. 2000; 543:241-3.

33. Kim H-J, Lee H-J, An SY, Sim S, Park B, Kim SW et al. Analysis of the prevalence and associated risk factors of tinnitus in adults. PLoS One. 2015;10(5).

34. Searchfield GD, Kobayashi K, Sanders M. An adaptation level theory of tinnitus audibility. Frontiers in Systems Neuroscience. 2012; 6(46).

35. Roberts LE, Husain FT, Eggermont FF. Role of attention in the generation and modulation of tinnitus. Neuroscience and Biobehavioral Reviews. 2013; 37:1754-1773.

36. Nascimento IP, Almeida AA, Diniz Junior J, Martins ML, Freitas TM, Rosa MR. Tinnitus evaluation: relationship between pitch matching and loudness, visual analog scale and tinnitus handicap inventory. Braz J Otorhinolaryngol. 2019; 85:611-616.
37. Lee JH, Ra J, Kim YH. Adequacy of the Simplified Version of the Tinnitus Handicap Inventory (THI-S) to Measure Tinnitus Handicap and Relevant Distress. Korean J Audio. 2014; 18:19-27.

38. Vasco O, Rute M, Nuno T-C. Correlatos de Ansiedade e Depressão nas queixas de acufenos. Braz. J. Hea. Rev. 2020; 3(3): 5818-5841.

39. Aazh H, Landgrebe M, Danesh AA, Moore BCJ. Cognitive Behavioral Therapy For Alleviating The Distress Caused By Tinnitus, Hyperacusis And Misophonia: Current Perspectives. Psychology Research and Behavior Management. 2019; 12: 991-1002.

\section{CORRESPONDÊNCIA}

Mariana Lopes Martins

Universidade Federal da Paraíba

Departamento de Fonoaudiologia

Centro de Ciências da Saúde.

Castelo Branco, João Pessoa, Paraíba, Brasil. CEP: 58051900

E-mail: mlopesmartins@hotmail.com 